\title{
Vivendo o Climatério: Percepção de mulheres usuárias de Unidade de Saúde da
}

\section{Família em Recife-PE}

\author{
Living the Climate Change: Perception of women users of the Family Health Unit in Recife-PE \\ Vivir el Climaterio: Percepción de las mujeres usuarias de la Unidad de Salud Familiar en Recife-
}

PE

Recebido: 25/11/2021 | Revisado: 30/11/2021 | Aceito: 04/12/2021 | Publicado: 14/12/2021

\author{
Virgínia Lima Pinto \\ ORCID: https://orcid.org/0000-0002-5481-4873 \\ Ponto Socorro Cardiológico de Pernambuco, Brasil \\ E-mail: ginalpinto@hotmail.com \\ Maria Carolina de Albuquerque Wanderley \\ ORCID: https://orcid.org/0000-0001-9373-8159 \\ Faculdade Medicina do Sertão, Brasil \\ E-mail: maria.wanderley@medicinadosertao.com.br \\ José Manoel Wanderley Duarte Neto \\ ORCID: https://orcid.org/0000-0002-3588-2934 \\ Faculdade Medicina do Sertão, Brasil \\ E-mail: jose.neto@medicinadosertao.com.br
}

\begin{abstract}
Resumo
O envelhecimento feminino representa um desafio de priorizar medidas de prevenção e promoção da saúde, e se destaca a atenção ao climatério e menopausa. O presente trabalho se propôs a conhecer e analisar o nível de informação das mulheres na fase do climatério, atendidas numa unidade de saúde da família (USF) de Recife - PE. Os dados foram obtidos através de entrevista estruturada aplicada após a consulta seguindo todas diretrizes de ética em pesquisa. As respostas das 20 voluntárias, de 45 a 79 anos, evidenciaram o perfil destas mulheres, os sintomas e problemas mais frequentes e quais as principais mudanças identificadas nesta fase. A maioria das mulheres relatou vivenciar ondas de calor e sintomas psicológicos, como mal-estar e irritação. Além de relacionarem a fase do climatério com sentimentos negativos. Foram evidentes a desinformação das usuárias e a necessidade de melhor suporte e orientação por parte dos profissionais de saúde. Ainda, ficou claro a importância de valorizar a influência da família sobre a adaptação, a ser considerada pela equipe multidisciplinar. Diversificadas foram as experiências relatadas, reafirmando que cada mulher vive esta fase de maneira individual, assim como deve ser a adequação de seu acolhimento, de forma individualizada, integrada e respeitosa.
\end{abstract}

Palavras-chave: Menopausa; Saúde da mulher; Saúde pública; Panorama; Fertilidade.

\begin{abstract}
Female aging represents a challenge to prioritize prevention and health promotion measures, and attention to menopause and climate change stands out. This study aimed to understand and analyze the level of information of women in the climacteric phase, attended at a family health unit (USF) in Recife - PE. Data were obtained through a structured interview applied after the consultation, following all research ethics guidelines. The answers of the 20 volunteers, from 45 to 79 years old, showed the profile of these women, the most frequent symptoms and problems and the main changes identified in this phase. Most women reported experiencing hot flashes and psychological symptoms such as malaise and irritation. In addition to relating the climacteric phase to negative feelings. The users' misinformation and the need for better support and guidance from health professionals were evident. Still, it was clear the importance of valuing the family over adaptation, what should be considered by the multidisciplinary team. Experiences reported were diversified, reaffirming that each woman lives this phase individually, as well as the adequacy of her reception should be, in an individualized, integrated and respectful way.
\end{abstract}

Keywords: Menopause; Women's health; Public health; Panorama; Fertility.

\section{Resumen}

El envejecimiento femenino representa un desafío para priorizar las medidas de prevención y promoción de la salud, destacando la atención a la menopausia y la menopausia. Este estudio tuvo como objetivo conocer y analizar el nivel de información de las mujeres en la fase climatérica, atendidas en una Unidad de Salud Familiar (USF) en Recife PE. Los datos se obtuvieron a través de una entrevista estructurada aplicada después de la consulta, siguiendo todas las pautas de ética de la investigación. Las respuestas de las 20 voluntarias, de 45 a 79 años, mostraron el perfil de 
estas mujeres, los síntomas y problemas más frecuentes y los principales cambios identificados en esta etapa. La mayoría de las mujeres informaron haber experimentado sofocos y síntomas psicológicos como malestar e irritación. Además de relacionar la fase climatérica con sentimientos negativos. La desinformación de los usuarios y la necesidad de un mejor apoyo y orientación por parte de los profesionales de la salud fueron evidentes. Además, quedó clara la importancia de valorar la influencia de la familia en la adaptación, para ser considerada por el equipo multidisciplinar. Las experiencias relatadas fueron diversificadas, reafirmando que cada mujer vive esta fase de manera individual, así como la adecuación de su recepción, de manera individualizada, integrada y respetuosa.

Palabras clave: Menopausia; Salud de la mujer; Salud pública; Panorama; Fertilidad.

\section{Introdução}

A expectativa de vida do brasileiro para 2050, está prevista para 80,75 anos. Esta transição demográfica traz consigo uma série de desafios que exigem a atenção de todos, principalmente do Estado no que se refere a implementação de políticas públicas (IBGE, 2018). Ainda, a feminização da terceira idade tem sido um fator preponderante nos estudos sobre envelhecimento (Camarano, 2003). É estimado que as mulheres vivem, em média, quase sete anos a mais que os homens e a projeção é que em 2050 haja 100 mulheres idosas para 76 idosos do sexo masculino (Lins e Andrade, 2018; WHO, 2015).

Com a idade, ocorrem mudanças na saúde. É crescente o número de mulheres climatéricas que procuram os serviços de saúde em busca de atendimento para tratar os desconfortos vivenciados nesse período. Elas sofrem diante das alterações físicas, psicossociais e culturais, dentre as quais: a perda da juventude, da atração física, da fertilidade e o declínio da sexualidade, causando medo, angústia e ansiedade (Silva, 2002). Sofrem também pela falta de um atendimento adequado, compreensivo e contínuo, em que possam manifestar as suas dificuldades (De Freitas, et al., 2004), fato agravado pela pequena qualificação dos profissionais, reforçada pela ausência de políticas públicas voltadas para o acolhimento e para a resolutividade desse tipo de queixa (Valença, et al., 2010).

O climatério, segundo Silva, Araújo \& Silva (2003), é definido como um período de transição entre os anos reprodutivos e não reprodutivos da mulher, que acontece na meia-idade. É caracterizado por alterações metabólicas e hormonais que, muitas vezes, podem trazer mudanças envolvendo o contexto psicossocial. Esse evento também pode ocorrer através de intervenção cirúrgica com a realização de ooforectomia bilateral associada, ou não, à histerectomia. É importante frisar que o climatério é um período abrangente da vida da mulher por não significar unicamente a última menstruação. A menopausa, marco desta fase, somente é reconhecida depois de 1 ano da sua ocorrência e acontece, geralmente, entre os 48 e os 50 anos (Brasil, 2008, Pinotti, et al., 1995). De modo geral, além das alterações nos sistemas endócrino, respiratório, circulatório e digestivo, os reflexos do tempo no corpo também se manifestam como perda de elasticidade da pele, perda de dentição, atrofia muscular, perda de movimento e aparecimento de doenças musculoesqueléticas. À medida que envelhecemos, novas doenças aparecem ou evoluem (Maximiano-Barreto, 2019).

Atendendo às reivindicações do movimento de mulheres, o Programa de Assistência Integral à Saúde da Mulher (PAISM), instituído pelo Ministério da Saúde, foi operacionalizado em 1984 (Brasil, 1984). Paralelamente, no âmbito do Movimento Sanitário, se concebia a estrutura conceitual que embasaria a formulação do Sistema Único de Saúde (SUS), onde se incluíam ações educativas, preventivas, de diagnóstico, tratamento e recuperação, englobando a assistência à mulher no climatério, além de tantas outras necessidades identificadas a partir do perfil populacional das mulheres (Brasil, 2011).

Embora já tenham se passado mais de 30 anos da formulação e implantação do PAISM, ainda é possível observar impasses no acompanhamento à saúde da mulher climatérica, notando-se uma deficiência na assistência, que não corresponde ao que se propôs o referido Programa, quando este inclui em seus preceitos ações educativas e preventivas de saúde, de forma que os cidadãos tenham conhecimento do seu estado de saúde ou doença (Vidal, 2009). Afinal, segundo Lopes (1998), a principal atitude profilática do climatério é promover o esclarecimento e o autoconhecimento. Uma pessoa que conheça seu próprio corpo e o ser, terá mais condições de se comportar de modo saudável, sem falsas expectativas, tabus ou receios 
fantasiosos, próprios de cada região.

Compreender a perspectiva das mulheres locais sobre sua experiência pode guiar um serviço de assistência mais adequado. Nesse contexto, o objetivo desta pesquisa foi de descrever o perfil sociodemográfico das mulheres que vivenciam o climatério, atendidas no mês de novembro de 2020, na unidade de Saúde da Família Severino Dias, do Distrito Sanitário VIII, no município de Recife-PE. Analisando, em paralelo, as suas experiências e o nível de informação dessas mulheres acerca desse período crítico de sua vida, para então compreender os significados das mudanças atribuídos por elas.

\section{Metodologia}

\subsection{Campo da Pesquisa}

Essa pesquisa foi realizada na Unidade de Saúde da Família Severino Dias (USF-SD), no bairro de Três Carneiros Baixo/Cohab, Distrito Sanitário VIII, Município de Recife - PE. Com 483,2 hectares e uma população residente de 69.134 habitantes, a Cohab é um bairro que apresenta, baixo Índice de Desenvolvimento Humano para cidade do Recife, sendo que em 2010 o seu IDH era de apenas 0,772 (IBGE, 2010). A Unidade de Saúde Severino Dias é constituída por 03 Equipes de Saúde da Família (ESF), sendo o estudo realizado na Equipe 01 da referida unidade de saúde.

\subsection{População Estudada}

A população de estudo foi constituída por 20 (vinte) mulheres na fase do climatério, atendidas no período de novembro de 2020, previamente aprazadas para a consulta no atendimento do Programa de Saúde da Mulher (PSM), na USFSD. A seleção dessa população atendeu os seguintes critérios: 1. Ser moradora da área atendida pela Equipe 01 da USF-SD; 2. Ser atendida no PSM da Equipe 01, da USF-SD; 3. Aceitar em participar da pesquisa e assinar o Termo de Consentimento Livre e Esclarecido (TCLE); 4. Responder questionário durante a consulta no PSM da USF-SD.

\subsection{Coleta de Dados}

Para a coleta dos dados foi elaborado um roteiro de entrevista estruturada, que foi utilizado durante as consultas com as mulheres, com a intenção de direcionar a conversa, identificando problemas e percepções da mulher com relação a essa fase de vida. O instrumento foi embasado nos trabalhos de: Vidal (2009), De Lorenzi \& Baracat (2005), com relação a qualidade de vida no climatério foi utilizado o Questionário de Saúde da Mulher (Women’s Health Questionnarie), um instrumento previamente validado para a língua portuguesa (Silva Filho, 2005) e, ainda, o questionário usado no Estudo de Correlação Analítica Hormonal da Menopausa (Menopause Analytical Hormonal Correlate Outcome Study - MAHCOS) (Braverman, et al., 2014).

O roteiro foi constituído de: identificação, idade, grau de escolaridade, estado civil, renda familiar e questões abertas enfocando o conhecimento sobre o climatério e abordando ainda as manifestações emocionais, metabólicas e sociais.

As entrevistas foram realizadas dentro do consultório médico, na USF-SD, durante as consultas individualizadas no PSM, que aconteceram as sextas-feiras, no período da manhã, por demanda espontânea. Onde, após a consulta, foi oferecido a essas mulheres o convite para participarem da pesquisa e, após informações, consentimento e preenchimento do TCLE, foi aplicado o questionário. A aplicação do instrumento foi realizada de forma individual e as respostas foram anotadas em sua integralidade.

\subsection{Aspectos Éticos}

A pesquisa cumpriu as exigências éticas fundamentais, descritos na Resolução nº 196/96 do CNS, de 10/10/1996 - do 
Conselho Nacional de Saúde - Ministério da Saúde que dispõe das Diretrizes e Normas Reguladoras de Pesquisa envolvendo seres humanos (Brasil, 1996). Todas as usuárias entrevistadas assinaram o TCLE, após as informações da pesquisa, onde foi assegurado o anonimato, assim as usuárias foram identificadas pelas suas iniciais. O projeto foi submetido e aprovado pelo Comitê de Ética em Pesquisa, através da Plataforma Brasil, com número do parecer: 4.339.025 e CAAE: 39046020.8 .0000 .5586 .

\subsection{Análise e Armazenamento dos Dados}

A análise e interpretação dos dados foram realizadas quantitativa e qualitativamente, e todas as tabulações e cálculos foram realizados em softwares Microsoft Office 365®. Todos os dados coletados foram armazenados em drive pessoal, na "nuvem", dos pesquisadores, além de um HD externo, para evitar qualquer perda de informação e disponibilizar esse material sempre que solicitado. O material será mantido pelo período mínimo de 5 anos após a conclusão do estudo.

\section{Resultados e Discussão}

\subsection{Perfil Socioeconômico}

Para a apresentação dos resultados desta pesquisa, inicialmente foram delineados de forma breve o perfil demográfico, social e econômico das mulheres participantes. A Tabela 1 apresenta o perfil das mulheres de acordo com faixa etária, estado civil, escolaridade, número de cômodos e renda familiar mensal.

O grupo estudado compõe-se de 20 mulheres entre 47 e 79 anos, que vivenciam a fase climatérica/menopausa; a faixa etária mais prevalente foi de 50 a 55 anos, com 35\%. Considerando a mudança no perfil dos usuários em saúde, no que diz respeito a envelhecimento e longevidade, onde estudos afirmam que até 2030 cerca de 1,2 bilhões de mulheres estarão na menopausa, anualmente esse número chegará a 47 milhões (Organização Pan-Americana de Saúde, 2013). Diante desse quadro os serviços de saúde precisam de uma maior organização e enfoque para atender a essa demanda.

Sobre o estado civil, 50\% das mulheres declararam ser casadas, $30 \%$ informam ser solteiras, $10 \%$ viúvas e as outras 10\% constituem união estável. Segundo Yang et al. (2016), o contexto familiar pode influenciar na forma como as mulheres vivenciam o climatério. Dentre as influências positivas, destacam-se o carinho, a afetividade e a relação de identidade como primordiais para o enfrentamento do estresse. As experiências compartilhadas pelas mulheres mais velhas da família amenizam a ansiedade trazida pelas expectativas acerca do novo. Entre as influências negativas, a falta de escuta por parte dos cônjuges pode causar conflitos. Lançar à mão estratégias que envolvam os homens no processo de cuidado das mulheres climatéricas é imprescindível para amenizar mal-entendidos (Caçapava, et al., 2016).

Já acerca da escolaridade, a maioria das mulheres entrevistadas declarou baixa escolaridade. 50\% possuem o ensino fundamental incompleto, $20 \%$ possuem o ensino fundamental completo, $15 \%$ têm o ensino médio completo, $5 \%$ têm o ensino médio incompleto e nenhuma possui o nível superior, ainda $10 \%$ são analfabetas. O que condiz com a realidade local do bairro estudado, com grande parcela da população em vulnerabilidade social.

Segundo Veloso, Maranhão \& Lopes (2013), a escolaridade influencia na percepção da mulher sobre esse período e torna-se importante para que possam entender e obter uma melhor preparação para enfrentar as diversas alterações que ocorrem nessa fase, uma vez que com certo nível de conhecimento torna-se mais fácil procurar e usar meios que as ajudem na manutenção de hábitos mais saudáveis, pois as mulheres irão vivenciar esse período por uma boa parte de sua vida.

Com relação a renda familiar, a pesquisa nos mostra que 55\% das mulheres possuem renda mensal menor que 01 salário-mínimo, que a outra metade (45\%) possui renda de 01-03 salários-mínimos. No Brasil, vivemos um processo de feminização da pobreza, além da velhice. As escassas informações acerca do climatério afetam mais as famílias de baixa renda, 
assim como foi visto no presente trabalho. Além disso, a baixa ou nenhuma escolaridade e a ausência de procura por serviços de saúde contribuem para a incompreensão da menopausa (Ferreira, et al., 2015).

\subsection{Causas do Climatério}

Durante a entrevista, foi questionado por qual o motivo as mulheres se encontravam na fase do climatério, se naturalmente pela idade (fisiologicamente) ou por outro motivo, seja por alguma doença ou por cirurgia. Na Tabela 2 foram dispostos os resultados encontrados: $55 \%$ das mulheres estavam na fase do climatério naturalmente, devido a idade, e as outras 45\% apresentaram outras causas. Os resultados de assemelham ao estudo de Mota et al. (2021), com mulheres do Sudoeste Baiano, onde 40,5\% das 42 entrevistadas relataram estar diagnosticadas com menopausa ou em estágio de climatério, já $31 \%$ declararam serem mulheres histerectomisadas (31\%).

\subsection{Reposição Hormonal}

O resultado da pesquisa revelou que nenhuma das usuárias entrevistadas fez uso de reposição hormonal. Tais resultados refletem uma mudança de paradigma de medicação, pois, antigamente, a assistência à mulher climatérica centravase principalmente na terapia de reposição hormonal. Entretanto, a partir de 2002, com a publicação dos resultados conhecidos por Women's Health Initiative (WHI), houve uma sensível redução na sua prescrição pois, os estudos apontam um risco significativamente maior de eventos tromboembólicos e câncer de mama, quando comparado ao grupo controle sem qualquer medicação hormonal. Ainda que, posteriormente, tais resultados tenham sido questionados por problemas metodológicos, acabaram por levar uma maior reflexão acerca da assistência à mulher climatérica (De Lorenzi e Baracat, 2005; Writing Group for the Women's Health Initiative Investigators et al., 2002; Lindsay, 2003).

As indicações da terapia hormonal tornaram-se restritas ao alívio dos sintomas vasomotores e da atrofia urogenital e na prevenção da osteoporose, não tendo sido esta última indicação referenda pelo FDA norte-americano (Sociedade Brasileira de Climatério - SOBRAC, 2003). Tal conservadorismo em relação à prescrição à terapia hormonal tem sido contestado por pesquisas recentes, cujos achados têm apontado para possíveis efeitos benéficos desta. Dentre estas, uma revisão do próprio estudo WHI demonstrou que a terapia hormonal teria um efeito de proteção cardiovascular quando iniciada nos primeiros dez anos após a menopausa, quando o processo de aterosclerose ainda é inicial (Mendelsohn et al., 2007).

Segundo a Sociedade Brasileira de Climatério, 75\% dos mais de 11 milhões de brasileiras com idades entre 45 e 64 anos sofrem com os efeitos do climatério e, dessas, $8 \%$ fazem tratamento com hormônios. Existem inúmeros fatores que estão relacionados com o desenvolvimento do câncer de mama. Sendo assim, é importante analisar a história reprodutiva de cada mulher, em qual idade ocorreu a primeira menstruação e início da menopausa, número de gestações. Esses são fatores relacionados ao estímulo estrogênico, que tem o risco aumentado conforme a exposição. Deve ser analisado também os hábitos e vícios, a história familiar e os fatores ambientais (INCA, 2019).

\subsection{Sintomas Relatados}

Conforme o exposto na Tabela 3, a maioria das mulheres entrevistadas apresentaram sintomas típicos do climatério/menopausa, umas de formas mais intensas, outras de forma mais leve, havendo ainda as que não apresentam nenhum sintoma. O sinal mais comum foram as ondas de calor ou fogachos, relatadas por $70 \%$ das usuárias. Os outros sintomas relatados apresentam relação psicológica: mal-estar, irritação/nervosismo e insônia. Apenas duas entrevistadas (10\%) relataram não sentir nada.

Em uma pesquisa qualitativa exploratório-descritiva realizada com 16 mulheres, em espaços públicos de Porto Alegre, RS, no ano de 2020, também foi constatado que os sintomas vasomotores foram os mais percebidos pelas entrevistadas e 
apenas três mulheres não referiram nenhum sintoma (Curta; Weissheimer, 2020). Já em outro estudo realizado no Rio Grande do Norte com 50 mulheres na fase do climatério, obteve-se os seguintes resultados com relação aos sinais e sintomas nessa fase: todas as respondentes apresentaram mais de um sintoma/sinal dentre os listados no roteiro de entrevista, caracterizando a síndrome do climatério, apresentada principalmente através de: artralgias/mialgias (74\%), ondas de calor (58\%), aumento da frequência miccional (50\%), cefaleias (54\%), pressão no cérebro e insônia (48\%) (Valença et al., 2010).

O mecanismo fisiopatológico das ondas de calor ainda não é totalmente conhecido, porém, sabe-se que o declínio dos níveis de estradiol interfere no centro regulador da temperatura localizado no hipotálamo, favorecendo a sua ocorrência. Caracterizam-se por uma sensação transitória e súbita de aumento da temperatura corporal, frequentemente acompanhada de sudorese, palpitações e cefaleia, que acaba por interferir nas atividades diárias e na qualidade do sono (Aldrighi et al., 2002; De Lorenzi e Baracat, 2005).

Dados de estudo realizado no sudoeste baiano mostra que, das 42 mulheres entrevistadas, a maioria tem observado instabilidade de humor e alterações no sono (aproximadamente 69\% e 61,9\%, respectivamente) (Mota et al. 2021). As modificações fisiológicas na mulher podem começar a surgir a partir dos 40 anos, podem perdurar até os 70 em casos mais raros. A depender da intensidade dos sintomas, poderão surgir transtornos físicos e/ou emocionais em determinados episódios do climatério, que afetarão sua qualidade de vida, podem fazer com que as mulheres vivenciem sentimentos de fracasso, perda da juventude, beleza e oportunidades (Hoffmann et al., 2015).

Assim como observado neste estudo, aproximadamente 50\% a 70\% das mulheres referem sintomas somáticos e dificuldades emocionais nos anos que seguem a menopausa, com destaque para ondas de calor ou fogachos, devido as usas implicações negativas para a sua qualidade de vida (Aldrighi et al., 2002; De Lorenzi e Baracat, 2005).

Como foi observado, as dificuldades com o sono são comuns em mulheres após os 50 anos, em especial a insônia, ainda que, até o momento, não tenha sido possível estabelecer uma associação definitiva destas com a queda estrogênica, sendo atribuídas, na maioria das vezes, à ocorrência de fogachos ou a dificuldades emocionais. Sabe-se que o despertar acompanhado de fogachos despende mais energia, resultando em fadiga, irritabilidade e até depressão (Souza e Aldrighi, 2001).

Embora não tenham sido relatados neste estudo, outras diferentes experiências são relatos comuns de mulheres no climatério. A alteração na voz, é um sintoma físico bastante relatado no climatério/menopausa, capaz de influenciar negativamente a qualidade de vida. Quanto mais a mulher perde a potência da sua voz, maior é a exteriorização do seu estresse, pois prejudica suas relações sociais (Basílio et al., 2016). A osteoporose é outra condição física comum na terceira idade, capaz de desestabilizar significativamente a qualidade de vida de mulheres em situação de climatério. Caracteriza-se por uma baixa massa óssea que deixa a mulher susceptível a fraturas (Costa et al., 2016).

A atrofia urogenital é mais uma das queixas comuns nessa fase e que pode causar intenso desconforto à mulher. Algumas poucas mulheres relataram esses desconfortos, como descrito no item 3.6. A intensificação do declínio estrogênico após a menopausa promove um adelgaçamento e enrijecimento da parede vaginal, que aliados a uma menor lubrificação, causam dispareunia e dificuldades no intercurso sexual. O terço distal da uretra sofre também um processo de atrofia, que se manifesta por disúria e urgência miccional (De Lorenzi e Baracat, 2005).

\subsubsection{Alterações Comportamentais}

No climatério, não são raras mudanças comportamentais, maior labilidade emocional e até dificuldades com a memória. Corroborando com a pesquisa segue falas das entrevistadas: "É uma coisa muito ruim na vida da gente, porque quando entra na menopausa sente muita coisa que não sentia." (C.M.S. - 79 anos). "[...]fiquei muito irritada [...]” (H.M.S. 76anos)

Não obstante, há dúvidas acerca do quanto tais queixas estariam relacionadas à queda estrogênica ou a fatores 
psicossociais e orgânicos relacionados ao processo de envelhecimento. O hipoestrogenismo interferiria na síntese dos neurotransmissores, modulando o comportamento e sintomas psíquicos. As próprias ondas de calor, dependendo da sua intensidade e frequência, por interferirem no sono e nas atividades cotidianas, seriam causa de irritabilidade e até depressão. Para outros autores, a maior ocorrência de depressão no climatério estaria relacionada principalmente ao medo de envelhecer aliado a sentimentos de inutilidade e carência afetiva (Dennerstein et al., 2002; De Lorenzi e Baracat, 2005).

As reações emocionais no climatério parecem ser particularmente influenciadas pelas atitudes em relação à menopausa, sendo menos intensas entre as mulheres que associam a menopausa à maior maturidade e autoconfiança. Todavia, para as mulheres que não conseguem redimensionar novas perspectivas existenciais, o climatério é acompanhado de sintomas mais intensos, maior irritabilidade, ansiedade, depressão e até dificuldades sexuais (Favarato, et al., 2000).

Não se pode desconsiderar também que, nas culturas ocidentais, a beleza física, a juventude e a maternidade são elementos de valorização da mulher, cuja perda pode favorecer sentimentos de desvalia, tristeza e até depressão. A maior percepção do envelhecimento e a intensificação da sintomatologia decorrente do hipoestrogenismo, aliados a eventos comuns nessa fase, como o crescimento dos filhos, a aposentadoria ou a morte do cônjuge demandam ajustes emocionais e mudanças de estilo de vida por vezes difíceis para a mulher. Ao mesmo tempo, cabe lembrar que, no climatério, as mulheres tendem a atribuir indiscriminadamente à menopausa tanto os sintomas relacionados à carência estrogênica, como as queixas físicas ou emocionais decorrentes de eventuais estados mórbidos prévios, confundindo-as (De Lorenzi e Baracat, 2005).

\subsection{Exercício Físico}

A atividade física é um recurso terapêutico valioso que deveria ser mais explorado nos anos do climatério. No presente trabalho, apenas 50\% das usuárias afirmaram realizar algum tipo de exercício físico, enquanto a outra metade afirmou não realizar nenhum exercício físico. No que tange à qualidade de vida, nas mulheres fisicamente ativas os sintomas climatéricos se mostram significativamente menos severos, o que reforça o papel positivo do exercício físico no climatério (Tairova e De Lorenzi, 2011).

Outro estudo de base populacional, realizado em Natal- RN com 370 mulheres entre 40 e 65 anos de idade, identificou um menor percentual dos sintomas intensos do climatério quando as mulheres eram ativas fisicamente (Gonçalves, 2011). Estudo do tipo caso-controle observou $63,6 \%$ das mulheres eram sedentárias, resultado semelhante ao encontrado no presente estudo. Elas apresentaram sintomas de intensidade moderada a severa no climatério e que há melhores escores no nível de qualidade de vida entre mulheres que realizavam atividade física regular (Tairova e De Lorenzi, 2011). Por isso a orientação sobre adoção de hábitos de vida saudáveis (prática regular de atividade física e alimentação adequada) pode representar uma mudança significativa na redução da intensidade dos sintomas e melhorar a qualidade de vida (Alves et al., 2015).

\subsection{Conceitos das Usuárias Sobre o Climatério}

O Quadro 1, reúne trechos de relatos das usuárias, apresentados em três categorias temáticas. As entrevistadas relataram a fase da menopausa com sentimentos negativos e como sintomas (sinônimo de calor e de mal-estar). Quando perguntadas sobre o climatério, a maioria desconhecia o significado da palavra, outras associavam menopausa como sinônimo de climatério. Isso pode ser explicado pelo fato das mulheres entrevistadas no presente trabalho apresentarem baixa ou nenhuma escolaridade.

Em estudo realizado no Rio Grande do Norte, com 50 mulheres na fase do climatério, $84 \%$ das mulheres disseram já ter ouvido falar sobre o climatério e $94 \%$ acerca do que é menopausa. No entanto, observou-se que a maior parte delas, ou seja $66 \%$, tinha uma visão inconsistente e superficial acerca do climatério, assim como observado neste trabalho (Valença et al., 2010). 
Isso revela que essas mulheres provavelmente não estão recebendo amparo, no que diz respeito as informações acerca de sua condição. Viver o climatério sem preparo pode gerar dificuldade para o enfrentamento dos desafios que surgem e, ainda, comprometer a autoestima, os relacionamentos e a qualidade de vida (Lomônaco et al., 2015).

Mulheres com uma percepção mais negativa da menopausa não somente tendem a apresentar uma pior qualidade de vida, como sintomas climatéricos mais severos as ondas de calor parecem ser os únicos sintomas diretamente associados com a queda estrogênica, ao passo que as demais queixas climatéricas (labilidade emocional, menor libido e insônia) estariam relacionadas principalmente ao modo como a menopausa é percebida (De Lorenzi e Baracat, 2005).

Quando compreendem os motivos de tais manifestações, menos intensos são os sintomas e menor é o impacto em suas vidas. Sendo bem orientadas sobre a menopausa, podem vivê-la de maneira menos sintomática, principalmente em relação aos sintomas psicológicos (Paiva et al., 2013). A percepção relatada pelas mulheres acerca das mudanças corporais e emocionais no climatério precisa ser escutada e compreendida, para que possam ser orientadas pelos enfermeiros, principalmente os de estratégia saúde da família, que desenvolvem consultas com ênfase na escuta e nas ações educativas (Santos et al., 2016).

Com relação a assistência prestadas pelos profissionais de saúde, as mulheres relataram que gostariam de receber mais informações a respeito do climatério/menopausa. Também afirmaram que gostariam de informações sobre práticas alternativas para aliviar os sintomas que aparecem nessa fase de vida. Frente ao exposto, é reconhecida a necessidade de se buscar um paradigma mais abrangente, não somente explicativo, mas sim interpretativo das questões relacionadas à saúde da mulher climatérica.

\subsection{Assistência Adequada em Saúde}

A assistência ao climatério está se expandido para além dos aspectos biológicos relacionados ao hipoestrogenismo, passando a considerar também a influência de fatores culturais e psicossociais, na busca de um cuidado mais integral e efetivo. Para tanto, é necessário que os profissionais de saúde procurem perceber a mulher climatérica na sua integralidade, individualizando as suas necessidades e disponibilizando tanto medidas de promoção à saúde, como terapêuticas e de reabilitação com vistas a proporcionar-lhe uma melhor qualidade de vida. Com isso se evitará a regulação indevida do corpo da mulher e a "medicalização" do envelhecimento feminino, abrindo um espaço para a participação da própria mulher nas decisões sobre o seu planejamento terapêutico (Gonçalves et al., 2003).

A despeito das várias possibilidades de intervenção no climatério, hoje se reconhece que para serem efetivas, devem ser sempre precedidas de uma escuta qualificada, que permita identificar as reais necessidades da mulher nessa fase, como foi observado nos resultados as mulheres entrevistadas apresentaram diferentes necessidades. É preciso que esta tenha espaço para manifestar a sua percepção e sentimentos acerca do momento que está vivenciando e as suas dificuldades pessoais, devendo ser informada sobre as mudanças que o seu corpo está sofrendo e as implicações para a sua saúde, como foi relatado por uma das entrevistadas que "deveria existir um lugar específico para a gente...". O climatério precisa ser entendido como um período normal de transição, em que a prevenção de doenças e o alívio de possíveis desconfortos podem ser abordados de diferentes maneiras (Berni et al., 2007).

A educação em saúde, em especial, tem merecido destaque, não somente por contribuírem para um maior autocuidado, como para a mudança de eventuais posicionamentos negativos acerca do envelhecimento feminino, esclarecendo dúvidas acerca do climatério e da menopausa e, consequentemente, reduzindo a ansiedade da mulher (De Lorenzi e Baracat, 2005). Como observado na fala da entrevistada: “...Deveria existir algo específico para gente, palestras, lugar para fazer exercícios e que trouxesse mais conhecimento para gente, porque as vezes a gente vai na internet, mas não sabe se a informação é segura." (M.J.S.- 54 anos). 


\section{Conclusão}

A chegada do climatério é algo que afeta consideravelmente a vida da mulher, pois ocorrem muitas modificações nesse período. Na maioria das vezes, a falta de informação ou de acompanhamento adequado contribui negativamente na qualidade de vida dessas mulheres. Através da presente pesquisa foi possível observar que o conhecimento da população estudada acerca da menopausa e do climatério são deficientes e/ou escassos. De modo geral, não houve distinção entre os sintomas no início do climatério ou da menopausa, o que geram dúvidas acerca da temática, o que revela um serviço de educação em saúde ainda incapaz de fornecer informação da maneira adequada às usuárias. Outro ponto importante é a valorização em se entender a influência da família, filhos ou cônjuges sobre o processo de envelhecimento e de adaptação à nova fase de vida. Nesse sentido, a equipe multidisciplinar deveria incluir os membros da família no processo de conhecimento e entendimento, acompanhamento dos sintomas transitórios que possam acometer a mulher nessa fase do climatério/ menopausa.

Foi possível perceber padrão de individualidade dos sintomas, ou seja, a sua personalidade, contribui para como cada mulher vive esta fase, seja de um jeito positivo ou negativo, mas sempre individual. Assim, é indicada a implementação de estratégias específicas para as mulheres nessa fase, pois esse período tem suas peculiaridades, que devem ser assistidas e acompanhadas, assim como os outros períodos da fase da vida das mulheres.

É relevante ainda o desenvolvimento de estudos futuros que abordem a temática no âmbito da gestão, a fim de expor aos gestores públicos a importância de criação de programas e capacitação dos profissionais de saúde para orientar as mulheres que vivenciam o climatério, oferecendo uma assistência direcionada, integral e de qualidade.

\section{Agradecimentos}

À Equipe da Unidade de Saúde da Família Severino Dias, em especial aos Agentes de Saúde e a todas as usuárias que participaram da pesquisa.

\section{Referências}

Aldrighi, J. M., Aldrighi, C. M. S., Aldrighi, A. P. S. (2002). Alteraçöes sistêmicas no climatério. Rev Bras Med. 59(esp):15-21.

Alves, E. R. P., Costa, A. M., Bezerra, S. M. M. S., Nakano, M. A. S., Cavalcanti, A. M. T. S., Dias, M. D. (2015). Climatério: a intensidade dos sintomas e o desempenho sexual. Texto Contexto Enferm, 24(1):64-71. doi: 10.1590/0104-07072015000590014

Basílio, B. N., Ribeiro, V. V., Pereira, E. C., Leite, A. P. D. (2016). Vocal self-assessment of women in menopause. Rev CEFAC, 18(3):649-55. doi: 10.1590/1982-0216201618312115.

Berni, N. I. O., Luz, M. H., Kohlrausch, S. C. (2007). Conhecimento, percepções e assistência à saúde da mulher no climatério. Rev Bras Enferm., 60(3):299306. doi: 10.1590/S0034-71672007000300010.

Brasil. (1984). Ministério da Saúde. Assistência Integral à Saúde da Mulher: bases da ação programática. Brasília, DF.

Brasil. (1996). Resolução no 196, de 10 de outubro de 1996. Dispõe sobre diretrizes e normas regulamentadoras de pesquisas envolvendo seres humanos. Diário Oficial [da] República Federativa do Brasil, 16 out. 1996. http://bit.ly/2ekI031.

Brasil. (2008). Ministério da Saúde. Secretaria de Atenção à Saúde. Departamento de Ações Programáticas Estratégicas. Manual de Atenção Integral à Saúde da Mulher no Climatério/Menopausa. Brasília: Editora do Ministério da Saúde.

Brasil. (2011). Ministério da Saúde. Secretaria de Atenção à Saúde. Departamento de Ações Programáticas Estratégicas. Política Nacional de Atenção Integral à Saúde da Mulher: Princípios e Diretrizes. 1. ed., 2. reimpr. Brasília: Editora do Ministério da Saúde.

Braverman, E. R., Han, D., Oscar-Berman, M., Karikh, T., Truesdell, C., Dushaj, K., et al. (2014). Menopause Analytical Hormonal Correlate Outcome Study (MAHCOS) and the Association to Brain Electrophysiology (P300) in a Clinical Setting. PLoS ONE, 9(9). doi: 10.1371/journal.pone.0105048

Caçapava, R. J. R., Quirino, B.C., Hoga, L.A.K., Rosa, P.L.F.S. (2016). Men's perceptions and attitudes toward their wives experiencing menopause. Journal of women \& aging, 28(4): 322-333. doi: 10.1080/08952841.2015.1017430

Camarano, A.A. (2003). Mulher idosa: suporte familiar ou agente de mudança? Estudos Avançados, 17(49). doi: 10.1590/S0103-40142003000300004 
Costa, A.L.D., Silva, M. A. C. N., Brito, L. M. O., et al. (2016). Osteoporosis in primary care: an opportunity to approach risk factors. Ver Bras Reumatol,. 56(2):111-116. doi: 10.1016/j.rbre.2015.07.014.

Curta, J. C., Weissheimer, A. M. (2020). Perceptions and feelings about physical changes in climacteric women. Revi Gaucha Enferm., 41(spe). doi: 10.1590/1983-1447.2020.20190198.

Dennerstein, L., Lehert, P., Guthrie, J. (2002). The effects of the menopausal transition and biopsychosocial factors on well-being. Arch Womens Ment Health, 5(1):15-22. doi: 10.1007/s007370200018.

Freitas, K. M., De Vasconcelos Silva, A. R., Da Silva, R. M. (2004). Mulheres vivenciando o climatério. Acta Scientiarum. Health Sciences, 26(1):121-128. doi: 10.4025/actascihealthsci.v26i1.1633.

Lorenzi, D. R. S., Baracat, E. C. (2005). Climatério e qualidade de vida. Femina, 33(12):899-903.

Favarato, M. E. C. S., Aldrighi, J. M., Fráguas Junior, R., Pires, A. L. R., Lima, S. M. R. R. (2000) Sexualidade e climatério: influência de fatores biológicos, psicológicos e sócio-culturais. Reprod. Clim, 15(4): 199-202.

Ferreira, I. C. C., Silva, S. S., Almeida, R. S. (2015). Menopausa, sinais e sintomas e seus aspectos psicológicos em mulheres sem uso de reposição hormonal. Ensaios Cienc, Cien Biol Agrar Saúde, 19(2):60-4.

Gonçalves, A. K. S., Canario, A. C. G., Cabral, P. U. L., Silva, R. A. H., Sprydes, M. H. C., Giraldo, P. C., Eleuterio Jr, J. (2011). Impacto da atividade física na qualidade de vida de mulheres de meia idade: estudo de base populacional. Rev Bras Ginec Obst., 33(12). doi: 10.1590/S0100-72032011001200006.

Gonçalves, R., Merighi, M. A. B., Aldrighi, J. M. (2003). Reflexões sobre o climatério com enfoque no corpo, na cultura e na subjetividade. Reprod. clim,, 18(1):108-12.

Hoffmann, M., Mendes, K. G., Canuto, R., Garcez, A. S., Theodoro, H., Rodrigues, A. D., Olinto, M. T. A. (2015). Padrões alimentares de mulheres no climatério em atendimento ambulatorial no Sul do Brasil. Ciência \& saúde coletiva, 20(5):1565-1574. doi: 10.1590/1413-81232015205.07942014.

IBGE - Instituto Brasileiro De Geografia E Estatística. (2018). Projeção da População. Rio de Janeiro. https://sidra.ibge.gov.br/tabela/1174

IBGE - Instituto Brasileiro De Geografia E Estatística. (2012). Censo Brasileiro de 2010. Rio de Janeiro: IBGE.

INCA - Instituto Nacional Do Câncer. (2019). Fatores de Risco para o Câncer de Mama. Ministério da Saúde, https://www.inca.gov.br/controle-do-cancer-demama/fatores-de-risco.

Mendelsohn, M. E., Karas, R. H. (2007). H. R. T. and the Young at Heart. The New England Journal of Medicine, 326(25):2639-2641. doi: 10.1056/NEJMe078072.

Mota, L. J., Matos, G. V., Amorim, A. T. (2021). Impactos do climatério em mulheres do sudoeste baiano. Research, Society and Development, 10(7). doi: $10.33448 /$ rsd-v10i7.16563.

Lindsay, R. What have we learned from clinical studies? Fractures and the interactions of bone mass and remodeling. Osteoporosis international, 14(5):S8-11. doi: 10.1007/s00198-003-1488-y.

Lins, I.L., Andrade, L.V.R. (2018). A feminização da velhice: representação e silenciamento de demandas nos processos conferencistas de mulheres e pessoas idosas. Mediações, 23(3):436-465. doi:10.5433/2176-6665.2018v23n3p436.

Lomônaco, C., Tomaz, R. A. F., De Oliveira Ramos, M. T. (2015). O impacto da menopausa nas relações e nos papéis sociais estabelecidos na família e no trabalho. Reprod. Clim., 30(2):58-66. doi: 10.1016/j.recli.2015.08.001.

Lopes, C. M. C. (1998). Atenção ao climatério: intervenções e preventivas. In: Halbe HW. Tratado de ginecologia. São Paulo: Roca, v. 1. cap. 17.

Maximiano-Barreto, M. A., Andrade, L., Campos, L. B., Portes, F. A., Generoso, F. K. (2019) A feminização da velhice: uma abordagem biopsicossocial do fenômeno. Interfaces Científicas - Humanas E Sociais, 8(2):239-252. doi: 10.17564/2316-3801.2019v8n2p239-252.

Organização Pan-Americana da Saúde. (2013). Organização Mundial da Saúde (OPAS/OMS Brasil). Envelhecimento ativo: uma política de saúde. Brasília.

Paiva, E. R., Silva, M. M., Oliveira, C. D. B., Leal, I. H. S., Araújo, V. S., Dias, M. D. (2013). Climateric manifestations most frequent among women of a family health unit. Rev. enferm. UFPE online, 7(11):6430-7. doi: 10.5205/reuol.3794-32322-1-ED.0711201316.

Pinotti, J. A., Halbe, H. W., Hegg, R. (1995). Menopausa. 1. ed. São Paulo. Roca

Santos, J. L., Leão, A. P., Gardengh, G. (2016). Disfunções sexuais no climatério. Reprod. Clim., 31(2):91. doi: 10.1016/j.recli.2016.08.001.

Silva, A. R. V. (2002). Sexualidade no climatério: vivências e sentimentos da mulher. 87p. Dissertação (Mestrado em Enfermagem) - Universidade Federal do Cerará, Fortaleza.

Silva Filho, C. R., Baracat, E. C., Conterno, L. O., Haidar, M. A., Ferraz, M. B. (2005). Climateric symptoms and quality of life: validity of women's health questionnaire. Revista de Saúde Pública, 39(3):333-9. doi: 10.1590/S0034-89102005000300002.

Silva, R. M., Araújo, C. B., Silva, A. R. V. (2003). Alterações biopsicossociais da mulher no climatério. Revista Brasileira em Promoção da Saúde, 16(2):2833. doi: 10.5020/18061230.2003.p28.

Sociedade Brasileira de Climatério (SOBRAC). (2003). Consenso brasileiro multidisciplinar de assistência à mulher climatérica. São Paulo: SOBRAC. 
Research, Society and Development, v. 10, n. 16, e375101623892, 2021

(CC BY 4.0) | ISSN 2525-3409 | DOI: http://dx.doi.org/10.33448/rsd-v10i16.23892

Souza, C. L., Aldrighi, J. M. (2001). Sono e climatério. Reprod Clim., 16(1):20-25.

Tairova, O. S., De Lorenzi, D. R. S. (2011). Influência do exercício físico na qualidade de vida de mulheres na pós-menopausa: um estudo caso-controle. Revista brasileira de geriatria e gerontologia, 14(1):135-145. doi: 10.1590/S1809-98232011000100014.

Valença, C. N., Nascimento Filho, J. M., Germano, R. M. (2020). Mulher no climatério: reflexões sobre desejo sexual, beleza e feminilidade. Saúde e Sociedade, 19(2):273-285. doi: 10.1590/S0104-12902010000200005.

Veloso, L. C., Maranhão, R. M. S., Lopes, V. M. L. V. (2013). Alterações biopsicossociais na mulher climatérica: uma revisão bibliográfica. Revista Interdisciplinar, 6, n. 3, p. 187-194, 2013

Vidal, C. R. P. M. (2009). Mulheres no climatério: desconhecimento, relacionamentos e estratégias. 102 p. Dissertação (Mestrado Acadêmico em Cuidados Clínicos em Saúde) - Universidade Federal do Ceará, Fortaleza.

Writing Group for the Women's Health Initiative Investigators et al. (2002). Risks and benefits of estrogen plus progestin in healthy postmenopausal women: principal results from the Women's Health Initiative randomized controlled trial. Jama, 288(3):321-333. doi: 10.1001/jama.288.3.321.

World Health Organization (WHO). (2015). World report on ageing and health. ISBN: 9789241565042.

Yang, C. F., Kenney, N. J., Chang, T. C., Chang, S. R. (2016). Sex life and role identity in Taiwanese women during menopause: A qualitative study. Journal of advanced nursing, 72(4):770-781. doi: 10.1111/jan.12866. 